\title{
El parto pretérmino: detección de riesgos y tratamientos preventivos
}

\author{
Fernando Althabe, ${ }^{1}$ Guillermo Carroli, ${ }^{2}$ Roberto Lede, ${ }^{3}$ \\ José M. Belizán ${ }^{1}$ y Omar H. Althabe ${ }^{1}$
}

RESUMEN Todos los años nacen en el mundo alrededor de 13 millones de niños prematuros. La mayor parte de esos niños nacen en países en desarrollo y constituyen el componente principal de la morbilidad y la mortalidad perinatales. En el presente estudio de revisión se analizaron los datos científicamente validados sobre las intervenciones que se emplean con la intención de evitar al menos una parte de los partos pretérmino y disminuir su impacto en la salud neonatal. Se consultaron las bases de datos Biblioteca Cochrane y Medline y se estudiaron 50 trabajos de revisión y artículos de investigación relacionados con el tema del parto pretérmino en sus siguientes aspectos: factores de riesgo y detección precoz del riesgo de parto pretérmino; prevención de la amenaza de parto pretérmino; tratamiento del parto pretérmino iniciado, y prevención del síndrome de dificultad respiratoria neonatal. Se encontraron pocos medios ensayados con éxito para predecir, prevenir o detectar precozmente la amenaza de parto pretérmino. Solo el tamizaje y tratamiento de la bacteriuria asintomática pueden recomendarse para todas las embarazadas como parte del control prenatal. El tamizaje de la vaginosis bacteriana y su tratamiento ulterior y el cerclaje profiláctico reducen, respectivamente, la incidencia de nacimientos adelantados en embarazadas con antecedentes de parto prematuro y en las que tienen antecedentes de más de tres partos pretérmino. Como tratamiento del parto iniciado antes de tiempo, con o sin rotura prematura de membranas, las intervenciones que han mostrado eficacia son la administración de betamiméticos a la parturienta para prolongar por 48 horas el período de latencia del parto y de indometacina con el mismo objetivo como medicamento de segunda elección. La administración prenatal de corticoides a la embarazada puede inducir la maduración pulmonar del feto y reducir el sindrome de dificultad respiratoria y la hemorragia ventricular, reduciendo así la mortalidad neonatal. Se recomienda continuar y apoyar las investigaciones básicas y epidemiológicas sobre la prevención para adquirir más conocimientos sobre las causas y mecanismos del parto pretérmino y cómo prevenir la morbilidad y mortalidad que produce.

Todos los años nacen en el mundo alrededor de 13 millones de niños pre-

\footnotetext{
1 Centro Latinoamericano de Perinatología y Desarrollo Humano. Toda correspondencia referente a este artículo debe enviarse a Fernando Althabe a la siguiente dirección: Hospital de Clínicas, piso 16, Casilla de correo 627, 11000 Montevideo, Uruguay. Correo electrónico: althabef@clap.ops-oms.org

2 Centro Rosarino de Estudios Perinatales. Rosario, Argentina.

3 Instituto Argentino de Medicina Basada en las Evidencias, Martínez, Argentina.
}

maturos. La mayor parte de esos nacimientos ocurren en países en desarrollo y contribuyen la proporción más extensa de la morbilidad y la mortalidad perinatales que se registran anualmente en todo el mundo (1). En los registros del sistema de salud público de la ciudad de Rosario, Argentina, la cifra correspondiente a nacimientos pretérmino -definidos como los que ocurren antes de la 37. a semana de la gestación - ha llegado hasta 78\% (Ca- rroli G., comunicación personal). La información originada en países industrializados revela valores similares, con nacimientos pretérmino que contribuyen de 69 a $83 \%$ de las muertes neonatales (2). Gran parte de la morbilidad perinatal grave también se asocia con esos nacimientos. En los nacidos pretérmino son mucho más frecuentes el síndrome de dificultad respiratoria, la enterocolitis necrotizante, la hemorragia intraventricular y disca- 
pacidades de largo plazo como la parálisis cerebral, la ceguera y la pérdida de la audición (2).

Por otra parte, el nacimiento pretérmino se relaciona con gastos importantes de salud pública. En los países industrializados, la mayoría de los niños con bajo peso al nacer suelen ser pretérmino. En un estudio llevado a cabo en los Estados Unidos de América, se estimó que los gastos adicionales en salud, educación y cuidados generales de niños de 15 años o menos que habían tenido bajo peso al nacer ascendieron a alrededor de US\$6 000 millones en 1988. Entre los nacidos con un peso menor de $1500 \mathrm{~g}$, que constituyen cerca de $1 \%$ de todos los nacimientos, el costo de la atención médica de cada niño durante el primer año de vida fue de $\$ 60000$ en promedio (3).

El cuadro 1 muestra las incidencias del parto pretérmino en distintas regiones del mundo. Contrario a lo que ocurre con el bajo peso al nacer, la incidencia del parto pretérmino no muestra diferencias importantes entre los países en desarrollo y los desarrollados.

Actualmente se reconoce que el parto pretérmino es un síndrome heterogéneo y que las estrategias para prevenirlo deben ser distintas de acuerdo con los subgrupos afectados. Existen por lo menos tres subgrupos del síndrome: a) casos asociados con la rotura prematura de membranas, b) casos debidos a aborto provocado o interrupción yatrógena de la gestación y c) casos espontáneos con membranas íntegras. Los tres tienen diferentes causas y pronósticos neonatales.

La rotura prematura de membranas se acompaña casi invariablemente de un nacimiento pretérmino, pero aún no está claro si en esos casos conviene aplicar medidas para demorar el inicio del trabajo de parto. Los casos que se producen por interrupción electiva de la gestación debido a complicaciones del embarazo solo pueden prevenirse atacando los estados patológicos fundamentales, tema que no se incluye en esta revisión. En el subgrupo de partos espontáneos, el trabajo de parto pretérmino se desencadena sin ninguna causa conocida que lo justifique. Este se considera potencialmente tratable

CUADRO 1. Incidencia de partos pretérmino en poblaciones de distintas regiones del mundo

\begin{tabular}{lccc}
\hline \multicolumn{1}{c}{ Región } & Años & $\begin{array}{c}\text { Parto } \\
\text { pretérmino }^{\text {a }}(\%)\end{array}$ & Tamaño muestral \\
\hline & & & \\
América Latina & $1981-1990$ & 7,7 & 507722 \\
África & $1981-1989$ & 9,9 & 47773 \\
Asia & $1979-1984$ & 9,3 & 94975 \\
Norteamérica & 1991 & 10,6 & 4513435 \\
Europa & $1976-1988$ & 5,9 & 13675 \\
Antigua URSS & 1986 & 9,2 & 74815 \\
Oceanía & $1984-1986$ & 5,8 & \\
\hline
\end{tabular}

Fuente: Ref. 1.

${ }^{a}$ Antes de la $37 .{ }^{\text {a }}$ semana de gestación.

con medidas dirigidas a evitar el inicio del trabajo de parto o detener su evolución.

La frecuencia relativa de cada uno de los subgrupos mencionados varía de acuerdo con la población. Los dos primeros se manifiestan con mayor frecuencia en poblaciones donde las condiciones de salud no son satisfactorias y la proporción de partos pretérmino que pueden tratarse por intervención médica es pequeña. Villar et al. (1) analizaron detenidamente ese aspecto y concluyeron que, de todos los partos pretérmino, podrían tratarse entre $37 \mathrm{y}$ $75 \%$. De estos, más de la mitad se inician después de las 35 semanas y no serían objeto de intervención. En consecuencia, entre 21 y $28 \%$ de los casos potencialmente tratables podrían beneficiarse de intervenciones oportunas. En cifras absolutas, esto significaría alrededor de 1,3 millones de nacimientos pretérmino anuales potencialmente tratables, de los cuales $75 \%$ ocurren en los países en desarrollo (1).

Los subgrupos que se consideran intratables lo son únicamente en función de la probabilidad de evitar la premadurez. En tales casos están indicadas todas aquellas intervenciones destinadas a disminuir la morbilidad y la mortalidad neonatales, como el uso de corticoides o la remisión a un centro de mayor complejidad.

Según los datos científicos disponibles, ciertas intervenciones pueden realizarse para evitar una parte de los partos pretérmino y disminuir su impacto en la salud neonatal. En el presente trabajo de revisión se analizó la información científica referente a la eficacia de esas intervenciones, de acuerdo con los aspectos siguientes: a) factores de riesgo y detección precoz de la amenaza de parto pretérmino; $b$ ) prevención de la amenaza de parto pretérmino; c) tratamiento de la amenaza de parto pretérmino; y d) prevención de la morbilidad y la mortalidad neonatales. El objetivo general de la revisión ha sido evaluar esa información y hacer recomendaciones para la práctica clínica y la salud pública, así como sugerir las áreas que requieren investigación.

\section{MATERIALES Y MÉTODOS}

Este trabajo consiste en una revisión de las principales intervenciones utilizadas actualmente para detectar, prevenir o tratar el parto pretérmino: la selección de embarazadas para programas de instrucción preventiva, el examen vaginal periódico, el monitoreo de la actividad uterina en el hogar, el apoyo social, el cerclaje cervical, la administración profiláctica de betamiméticos, la administración de suplementos nutricionales, la administración de progesterona, la administración de antibióticos y el tratamiento con agentes tocolíticos. La mayor parte de las intervenciones seleccionadas habían sido evaluadas en ensayos clínicos contro- 
lados. A ese grupo se agregaron dos intervenciones (la selección por factores de riesgo y la detección de la fibronectina cervical) que, a pesar de no cumplir con el criterio anterior, se consideró importante comentar. También se agregó información sobre la prevención del síndrome de dificultad respiratoria neonatal.

La efectividad de cada una de las intervenciones seleccionadas se analizó sobre la base de revisiones sistemáticas de los ensayos clínicos controlados en los que se pusieron a prueba esas intervenciones (4). Con respecto a las intervenciones no sometidas a revisiones sistemáticas, la evaluación se basó en los informes publicados de las investigaciones clínicas originales. Se evaluaron 10 ensayos clínicos controlados sobre cinco intervenciones que no habían sido incorporados a las revisiones sistemáticas por haberse publicado posteriormente, y tres estudios sobre el tacto vaginal periódico y el monitoreo de las contracciones uterinas en el hogar, dos intervenciones que no se habían revisado.

Como fuentes de datos de revisiones sistemáticas se usaron la Cochrane Database of Systematic Reviews [Base de Datos Cochrane de Revisiones Sistemáticas], la Database of Abstracts of Reviews of Effectiveness (DARE) [Base de Datos de Resúmenes de Revisiones sobre Efectividad] incluida en una cuarta versión en disco (Disk Issue 4) de la biblioteca electrónica Cochrane (4) y la segunda versión en disco de la Cochrane Pregnancy and Childbirth Database 1995 [Base de Datos Cochrane sobre el Embarazo y el Parto 1995] (Disk Issue 2) (5).

Como fuente de datos principal de ensayos clínicos controlados se usó el Cochrane Controlled Trials Register [Registro Cochrane de Ensayos Controlados] incluido en la misma cuarta versión en disco de la biblioteca electrónica Cochrane $(5,6)$. La base de datos electrónica MEDLINE se empleó para actualizar la búsqueda con trabajos publicados de enero a noviembre de 1998. Se usaron los encabezamientos MeSH labor [parto] y premature [prematuro], acompañados de randomized control trial [ensayo controlado aleatorizado], meta-analysis [metanálisis] y review [revisión] en cuanto a tipo de publicación. Los puntos finales o resultados considerados fueron la incidencia del parto pretérmino, la mortalidad perinatal o neonatal y variables de morbilidad neonatal como la dificultad respiratoria y la hemorragia intraventricular.

La búsqueda bibliográfica fue realizada por uno de los autores (FA) y verificada mediante la selección al azar de intervenciones por un segundo autor (GC).

Dada la continua publicación de estudios, algunas de las revisiones analizadas están desactualizadas. No se realizó una actualización de las mismas para no interferir con el proceso de la Colaboración Cochrane, pero sí se analizaron los nuevos estudios publicados. Cuando se creyó conveniente, los autores incluso comentaron en detalle los estudios originales comprendidos en una revisión.

Los resultados de las revisiones sistemáticas se analizaron mediante metanálisis y se presentaron como riesgos relativos (RR) o razones de posibilidades (RP, odds ratio en inglés), de acuerdo con la presentación original de la revisión y sus intervalos de confianza de $95 \%$, presumiendo un modelo de efecto fijo (fixed effect model). Los resultados de los ensayos clínicos aleatorizados que se comentan se expresan como RR o RP con sus respectivos intervalos de confianza (IC) de 95\% y se consideran estadísticamente significativos si ambos límites del IC muestran un efecto en un mismo sentido, es decir, si son ambos mayores o menores de 1 .

En el caso de las pruebas sobre la capacidad diagnóstica de la selección por factores de riesgo y la determinación de fibronectina fetal, se presentan las capacidades operativas (sensibilidad, especificidad y valor predictivo). La capacidad diagnóstica de la detección de fibronectina fetal se investigó también mediante revisiones sistemáticas de métodos diagnósticos $(14,15)$.

Se consideraron en total 25 intervenciones de las cuales 21 fueron evaluadas en ensayos clínicos controlados y 20 están incluidas en 32 revisiones sis- temáticas (23 de la biblioteca Cochrane y 9 publicadas en revistas científicas).

\section{RESULTADOS}

\section{Factores de riesgo y detección precoz de la amenaza de parto pretérmino}

Con objeto de prevenir los nacimientos prematuros y las complicaciones que con ellos se asocian, a menudo se ha intentado identificar a las embarazadas en alto riesgo de parto pretérmino. Algunos factores de riesgo son bien conocidos: antecedentes de parto pretérmino o aborto tardío, embarazo múltiple, bajo peso antes del embarazo, aumento insuficiente del peso corporal durante el embarazo, edad menor de 20 ó mayor de 35 años, ocupación que requiere esfuerzo físico intenso o estar de pie durante largos períodos y nivel socioeconómico bajo $(1,2)$.

Se han ideado sistemas de puntuación basados en estos factores y otros, con el propósito de planificar acciones preventivas o terapéuticas para los grupos con puntuaciones elevadas (7), pero su ejecución se ha visto obstaculizada por varios problemas. En primer lugar, los sistemas ideados mostraron baja sensibilidad (menor de 50\%) en la práctica y valores predictivos positivos entre 17 y $34 \%$, especialmente en mujeres primigestas. El segundo factor es que, hasta donde se sabe, no hay ensayos controlados en los que se hayan comparado muestras de embarazadas que reciban atención prenatal sobre la base de selección por riesgo con otras en las que el control prenatal se realice sin ese enfoque. En consecuencia, no se cuenta con pruebas fiables de que la atención especial basada en factores de riesgo sea efectiva. Por último, cabe observar que con frecuencia no están bien evaluadas las intervenciones que se aplican a mujeres que se consideran de alto riesgo y es posible que produzcan más daños que beneficios. Cuando el enfoque de riesgo se aplica a la práctica clínica, existe el peligro de que el riesgo impreciso de un mal resultado sea reemplazado por tratamientos e intervenciones cuyos beneficios no 
han sido demostrados y cuyos riesgos son desconocidos (7).

Educación para la prevención de los partos pretérmino. Una estrategia para intentar reconocer tempranamente a las gestantes con una probabilidad aumentada de tener un parto pretérmino consiste en los programas de instrucción para las embarazadas y su familia. Se enseña a reconocer pronto las contracciones uterinas prematuras y la importancia de un diagnóstico precoz (8). En una revisión sistemática publicada en 1995, se evaluó el efecto en varios resultados perinatales de programas de educación para prevenir los partos pretérmino. En cinco investigaciones que incorporaron un total de 5778 pacientes, no se encontraron diferencias en la incidencia de parto pretérmino (<37 semanas) $(\mathrm{RP}=1,08$; IC95\%: 0,92 a 1,27) ni en la supervivencia neonatal $(\mathrm{RP}=1,00$; IC95\%: 0,99 a 1,01) (9). Los autores puntualizan que si bien no se demostraron efectos beneficiosos, hubo algunas limitaciones en el análisis efectuado: las pacientes incluidas en los estudios tenían alto riesgo de parto prematuro. Ello permite especular sobre su uso en pacientes con bajo riesgo, en quienes el efecto pudiera ser beneficioso. Esta interrogante debería ser evaluada por medio de un ensayo apropiado.

Examen vaginal periódico. En el cuello uterino pueden ocurrir modificaciones algún tiempo antes de desencadenarse el trabajo de parto pretérmino, por lo que, a manera de hipótesis, podría aducirse la utilidad del tacto vaginal periódico para identificar a aquellas mujeres en alto riesgo de padecerlo. Sin embargo, esta práctica tiene poco valor predictivo y a su vez puede ocasionar efectos adversos, como la rotura prematura de membranas o el inicio de las contracciones uterinas (6). Una investigación llevada a cabo en siete países europeos con objeto de evaluar la eficacia del tacto vaginal periódico en más de 5000 embarazadas no mostró ningún beneficio en función de la inciden- cia de partos pretérmino o muertes perinatales (10).

\section{Monitoreo de la actividad uterina en} el hogar. Este monitoreo es un sistema de detección precoz del trabajo de parto pretérmino, que consiste en el registro hogareño de las contracciones uterinas por medio de tocodinamómetros y la transferencia telefónica de la información recolectada a un centro de salud. La hipótesis es que antes del desencadenamiento del trabajo de parto ocurren contracciones anormales que no son advertidas por la mujer. Los tocodinamómetros son dispositivos electrónicos que identifican estas contracciones, lo cual permite la administración temprana de inhibidores. De las investigaciones publicadas sobre este tema, las más importantes, tanto por su calidad metodológica como por el número de pacientes seleccionados por métodos aleatorios, son dos estudios colaborativos multicéntricos realizados en los Estados Unidos de América $(11,12)$. Entre ambos se incluyeron más de 3500 pacientes embarazadas con alto riesgo de parto pretérmino. Ninguno de los dos muestra una disminución significativa de la incidencia de parto pretérmino o de la morbilidad o mortalidad neonatal.

Detección de la fibronectina. La fibronectina fetal es una glucoproteína presente en el líquido amniótico, la placenta y la membrana decidua, que puede liberarse en el flujo cervical o vaginal por un daño mecánico o debido a inflamación de las membranas o la placenta. Hay quienes consideran que la presencia de esta sustancia en el flujo cervical o vaginal es una prueba útil para identificar a las embarazadas en riesgo de parto pretérmino, dentro de la población que consulta por hipercontractilidad con membranas íntegras. Un estudio de Lockwood et al. mostró que en el grupo de embarazadas con una prueba positiva a la presencia de fibronectina, $83 \%$ tuvieron un parto pretérmino, en comparación con $19 \%$ de las mujeres con resultados negativos (13). Sin embargo, cuando esa prueba se utiliza como forma de tamizaje entre la 24. a y la 36. ${ }^{\text {a }}$ semanas de gestación en pacientes asintomáticas, su capacidad predictiva no es tan clara. Se han publicado recientemente dos revisiones sistemáticas de estudios sobre ese método $(14,15)$, que se basan en investigaciones de buena calidad metodológica. Los resultados indican que la presencia de fibronectina fetal en el flujo cervical o vaginal tiene un valor limitado para predecir el parto pretérmino, ya que los resultados positivos y negativos de la prueba generan cambios de mínimos a moderados en la probabilidad previa de parto pretérmino, tanto en pacientes de bajo como de alto riesgo. Por lo tanto, aunque es el principal marcador bioquímico disponible para identificar casos potenciales de parto pretérmino, no tiene buena capacidad predictiva $\mathrm{y}$, hasta el momento, no se ha evaluado en investigaciones clínicas aleatorizadas asociadas con intervenciones preventivas eficaces.

Apoyo social. La incidencia de parto pretérmino suele ser más alta en las mujeres de clase socioeconómica baja que en las de clase alta (2). Las razones no se conocen en detalle pero es probable que haya muchos factores involucrados. Partiendo de la hipótesis de que algunos de los factores son atribuibles al estrés, la falta de apoyo afectivo y la falta de conocimientos y acceso a la información, se llevaron a cabo varias investigaciones controladas. En ellas se compararon las intervenciones que intentaban solucionar o atenuar el impacto de los factores mencionados con la atención prenatal habitual. Lamentablemente, las intervenciones no resultaron efectivas para reducir la incidencia del parto pretérmino (16). La revisión sistemática de la Biblioteca Cochrane comenta lo siguiente sobre el tema: "Las mujeres embarazadas necesitan y desean tener ayuda y apoyo de sus familias, amigos y profesionales de la salud. Sin embargo, ese apoyo no es suficiente para revertir los efectos de toda una vida de pobreza y desventajas y por ende influir el curso de la gestación. Las embarazadas y quienes cui- 
dan de ellas deben estar informados de que los programas que ofrecen apoyo adicional durante el embarazo probablemente no reduzcan las probabilidades de un parto pretérmino".

Cerclaje cervical. El cerclaje se utiliza en casos de sospecha de incompetencia ístmico-cervical y puede que sea eficaz en los casos diagnosticados acertadamente. Sin embargo, el diagnóstico de incompetencia es muy difícil y con frecuencia inexacto. Una investigación multicéntrica europea (17) evaluó el efecto del cerclaje en pacientes cuyo médico dudaba de la utilidad del procedimiento en ese caso en particular. Los resultados muestran que la intervención solo disminuye la incidencia del parto pretérmino en mujeres con antecedentes de tres o más partos pretérmino y que se asocia con una frecuencia aumentada de hospitalización, infección, uso de agentes tocolíticos, inducción del parto y operación cesárea.

Administración profiláctica de betamiméticos. En pacientes con alto riesgo de parto pretérmino o que consultan por contracciones uterinas durante el embarazo, la administración de agentes betamiméticos por vía oral es una práctica profiláctica muy difundida entre los obstetras. En Holanda y partes de Bélgica, 45\% de los obstetras realizan esa intervención (2). En nuestra región no se conocen las cifras, pero no dudamos de que superan holgadamente a las citadas.

Los datos provenientes de investigaciones controladas (18) muestran que los betamiméticos no tienen ningún efecto beneficioso sobre el resultado perinatal. Si bien es cierto que hacen falta investigaciones de mejor calidad y mayor tamaño muestral para concluir la evaluación, hasta el momento no hay pruebas que justifiquen la utilización de esos agentes en la práctica clínica.

\section{Tratamiento con suplementos nutricionales}

El estado nutricional materno previo y la ganancia de peso durante el embarazo están fuertemente asociados con el crecimiento fetal, pero tienen poca relación con la duración del embarazo $(19,20)$. Esta asociación entre los datos antropométricos maternos y los resultados perinatales proviene de estudios con diseños observacionales (21); las pruebas experimentales provienen de investigaciones clínicas sobre la suplementación calórica y proteínica de la dieta de las embarazadas. Esos estudios están analizados en varias revisiones de la Biblioteca Cochrane (22-24). De 13 investigaciones sobre la suplementación calórico-proteínica equilibrada que allí se han revisado, solo en cinco se midió el efecto en el parto pretérmino. Los resultados indican un leve efecto protector en los grupos que recibieron los suplementos (RR: 0,83; IC95\%: 0,65 a 1,06).

Recientemente se publicó en Gambia un estudio de una población de mujeres embarazadas mal nutridas (25), que no está incluido aún en la revisión. El grupo cuya dieta se suplementó con galletas de alto contenido calórico en la segunda mitad de la gestación tuvo una incidencia menor de niños con bajo peso al nacer que el grupo control que no recibió el suplemento, pero la incidencia del parto pretérmino no se modificó.

Suplementación con magnesio. La administración rutinaria de magnesio durante el embarazo fue objeto de varios ensayos controlados, basados en los resultados de estudios observacionales que señalaban que las embarazadas con alta ingesta de este mineral tenían recién nacidos con mayor peso. De los cinco estudios incluidos en una revisión de la Biblioteca Cochrane, solo uno tiene buena calidad metodológica (26), de modo que los resultados globales, que orientan hacia un posible efecto beneficioso (RR: 0,75; IC95\%: $0,58$ a 0,96$)$, no son confiables. Por lo tanto, aún no existe ninguna confirmación que claramente avale su indicación rutinaria en función de prevenir el parto pretérmino, pero es importante que se realicen investigaciones apropiadas para aclarar la efectividad de esta intervención (27).

Suplementación con calcio. Hasta el momento tampoco se ha dilucidado el papel del calcio en la prevención del parto pretérmino. La suplementación con calcio se ensayó para validar la hipótesis de que reduce la incidencia de preeclampsia, pero en varias de las investigaciones publicadas se observa que reduce la incidencia del parto pretérmino. Tres revisiones sistemáticas sobre el tema indican que, aunque hay resultados que sugieren la eficacia de una mayor ingesta de calcio durante el embarazo para prevenir el parto pretérmino en ciertos grupos de pacientes con alto riesgo de hipertensión, todavía faltan investigaciones que validen esos resultados para que la intervención sea recomendable (28-30). En conclusión, la suma de todas las pruebas disponibles todavía no avala la suplementación sistemática con calcio para la profilaxis del parto pretérmino.

Suplementación con cinc. En varios estudios observacionales se ha registrado una asociación entre concentraciones bajas de cinc en la sangre materna y un mayor riesgo de parto pretérmino o de bajo peso al nacer $(31,32)$. A partir de estos hallazgos se han realizado varias investigaciones controladas que probaron que el efecto de la suplementación con cinc durante el embarazo (La Biblioteca Cochrane analizó cuatro de ellas, donde se mide la incidencia de parto pretérmino y se muestra una disminución de 24\% (RR: 76; IC95\%: 0,53 a 1,10) no es estadísticamente significativo (32). Uno de esos estudios, por Goldenberg et al. (33), es distinto del resto porque incluyó solo a mujeres con bajas concentraciones plasmáticas de cinc. Los resultados de esta investigación son similares al resto de los incluidos en la revisión Cochrane (RR: 0,77; IC95\%: 0,49 a 1,20).

Una investigación escandinava de reciente publicación (34) no se incluyó en la revisión. Sus resultados no muestran un efecto sobre la incidencia del parto pretérmino, pero el estudio no 
es del todo fiable desde el punto de vista metodológico, ya que excluye a $40 \%$ de las mujeres que participaron por falta de cumplimiento. Es importante aclarar el papel de la suplementación con cinc en la prevención del parto pretérmino por medio de una buena investigación que incluya a pacientes con deficiencias de este micronutriente.

Suplementación con hierro y folatos. La suplementación con hierro y folatos durante el embarazo ha sido motivo de múltiples ensayos controlados, pero en muy pocos se ha medido el efecto sobre el parto pretérmino. Solo un estudio de una población bien nutrida informa de los efectos de la suplementación con hierro en la incidencia del parto pretérmino y muestra una tendencia protectora (RR: 0,71; IC95\%: 0,48 a 1,06) (35). En cambio, el efecto de la suplementación con folatos sobre el parto pretérmino fue notificado en cuatro investigaciones analizadas en una revisión de la Biblioteca Cochrane (36), que no mostró ningún efecto beneficioso.

Pese a que está bien documentado el efecto beneficioso de la suplementación con hierro y folatos sobre la anemia materna en mujeres con deficiencias nutricionales $(36,37)$, hasta el momento no hay pruebas que indiquen claramente una reducción de la incidencia de partos pretérmino por efecto de esa intervención.

\section{Administración de progesterona}

Los agentes progestacionales se han recomendado para la profilaxis del parto pretérmino por su actividad relajante de la musculatura lisa uterina. En una revisión sistemática de la Biblioteca Cochrane (38) se incluyen seis investigaciones que evalúan la intervención y muestran que produce una reducción de casi $50 \%$ en la incidencia del parto pretérmino (RP: 0,53; IC95\%: $0,27$ a 1,05$)$, resultado que no es estadísticamente significativo. A pesar de su tendencia a reducir el parto pretérmino, la progesterona no reduce la morbilidad y mortalidad perinatales.
El número total de pacientes estudiados no permite sacar conclusiones definitivas. La última actualización de esta revisión sistemática se realizó a fines de 1993 y no tenemos conocimiento de que haya más publicaciones relevantes sobre el tema. Es importante planificar nuevas investigaciones de mayor tamaño muestral que evalúen la efectividad de la progesterona micronizada, que puede aplicarse por vía oral o vaginal y que no estaba disponible en el momento en que se realizaron las investigaciones analizadas.

\section{Administración de antibióticos}

Se ha validado científicamente que el tratamiento con antibióticos de pacientes embarazadas que padecen bacteriuria asintomática reduce la probabilidad de un parto pretérmino (39). No se conoce el mecanismo preciso que causa este efecto, pero se supone que puede deberse a la disminución de la incidencia de pielonefritis o de la colonización bacteriana vaginal por agentes patógenos como los estreptococos del grupo B. Una revisión de la Biblioteca Cochrane analiza 10 estudios que midieron el efecto del tratamiento con antibióticos sobre la incidencia del parto pretérmino o de nacidos de bajo peso. Los resultados muestran una reducción de $36 \%$ en la incidencia de ambos (RR: 0,64; IC95\%: 0,50 a 0,82 ). Villar et al. identificaron aquellos estudios incluidos en la revisión que indicaban separadamente el efecto sobre el parto pretérmino y registraron un resultado similar (RR 0,53; IC95\%: 0,33 a 0,66) (35).

La colonización vaginal por las bacterias anaerobias Gardnerella vaginalis o Mycoplasma hominis constituye un cuadro llamado vaginosis bacteriana. Se han publicado varios estudios de observación que muestran una asociación entre la vaginosis y el parto pretérmino. Hay et al. (40) observaron una asociación moderada (RR: 2,8; IC95\%: 1,1 a 7,4 ) en 783 embarazadas, mientras que Hillier et al. (41) señalaron que el efecto protector de los antibióticos había sido menor en una cohorte de 10000 pacientes (RR: 1,4; IC95\%: 1,1 a
1,8). Se especula que el mecanismo por el cual la infección vaginal contribuye al parto pretérmino es la infección ascendente subclínica de las membranas, la cual desencadena un proceso inflamatorio que a su vez provoca el trabajo de parto o la rotura prematura de las membranas (2). Si el tratamiento con antibióticos fuera eficaz contra la vaginosis y se administrara antes del desencadenamiento del proceso intrauterino, podría reducir la incidencia del parto pretérmino.

Una investigación aleatorizada demostró la eficacia del tratamiento de la vaginosis con metronidazol, que la erradicó en $87 \%$ de los casos tratados (42). En otra investigación realizada en mujeres con antecedentes de parto pretérmino o un peso corporal menor de $50 \mathrm{~kg}$ antes del embarazo, se administró de forma aleatoria una combinación de metronidazol y eritromicina por vía oral o placebo entre las 22 y 24 semanas de gestación. El tratamiento se repitió de dos a cuatro semanas después en las pacientes que siguieron teniendo vaginosis. Según los resultados, en las mujeres con vaginosis en alto riesgo de parto prematuro, este riesgo se redujo significativamente (43). Una revisión sistemática reciente que incluye cinco investigaciones sugiere que ese sería el grupo de pacientes beneficiado (44). Es importante tener en cuenta que aún no hay pruebas fiables de que el tratamiento antibiótico reduzca la proporción de partos pretérmino en todas las embarazadas con vaginosis, por lo cual todavía no está indicado el tamizaje de pacientes en bajo riesgo de parto pretérmino.

\section{Tratamiento de la amenaza de parto pretérmino con agentes tocolíticos}

Administración de betamiméticos. Estos agentes, que se administran a menudo a la embarazada en riesgo inminente de parto pretérmino, han sido objeto de evaluación frecuente. Las investigaciones de tamaño muestral y diseño apropiados (45), así como los metanálisis sobre el tema (46), muestran que los betamiméticos reducen de manera significativa el número de em- 
barazadas que tienen el parto en las 24 a 48 horas posteriores al inicio del tratamiento, pero tienen solo un efecto marginal en la reducción del parto antes de la 37. ${ }^{\text {a }}$ semana. Además, su uso no se asocia con variaciones de la morbilidad y la mortalidad perinatales. Esto ha generado controversias en torno a si deberían utilizarse de manera rutinaria y se ha concluido que, si bien los betamiméticos prolongan el embarazo solo por corto tiempo (24$48 \mathrm{~h}$ ), ese intervalo puede aprovecharse para realizar intervenciones que mejoren la supervivencia del neonato (2): administración de corticoides para promover la madurez pulmonar fetal y traslado de la madre a un centro de complejidad suficiente para atender un parto pretérmino (46).

Otro motivo para ser muy cautelosos al administrar los betamiméticos es que no son inocuos para la parturienta. En un ensayo llevado a cabo en el Canadá, más de $50 \%$ de las pacientes que recibieron ritodrina tuvieron efectos adversos, desde palpitaciones hasta un caso de edema pulmonar (45). Por este motivo se discute la edad gestacional límite para su utilización. En los países industrializados, el límite superior se

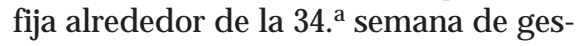
tación (dado que la mortalidad neonatal es muy baja $[<1 \%]$ pasado ese punto) y el límite inferior en la 24 . $^{\text {a }}$ semana, por lo baja que es la supervivencia neonatal antes de esa edad gestacional (2). En América Latina y el Caribe no pueden considerarse automáticamente los mismos límites, ya que la calidad de la atención perinatal varía de un país a otro y aun dentro de cada país. El límite superior podría ser un poco bajo en varias regiones donde existe la necesidad imperiosa de remitir a las parturientas a centros de mayor complejidad, aun cuando el trabajo de parto se inicia en la 34 . $^{\mathrm{a}}$ ó 35 . $^{\mathrm{a}}$ semana de la gestación. No tenemos conocimiento de investigaciones locales científicamente rigurosas que den respuesta a estas interrogantes.

Se están investigando otras formas de administrar los agentes betamiméticos, con el fin de aumentar su eficacia y disminuir los efectos adversos. Su administración en microinyecciones y su infusión en forma de microémbolos han mostrado igual capacidad para inhibir las contracciones uterinas con menores dosis y menos efectos adversos. Todavía hacen falta más ensayos controlados antes de poder recomendar su uso regular en la práctica clínica $(2,47)$.

Betamiméticos para mantenimiento. Una práctica habitual en los servicios obstétricos es la administración por vía oral de agentes betamiméticos para mantenimiento, después de haberse detenido la amenaza inicial de parto pretérmino. En un trabajo de revisión sistemática en que se analizan las investigaciones sobre esta intervención, se concluye que no proporciona ningún beneficio en función de la incidencia de parto pretérmino, la recurrencia de las contracciones uterinas o un aumento del intervalo antes del parto (48). Dos ensayos controlados con placebo - más recientes y, por lo tanto, no incluidos en la revisión mencionadaarrojaron resultados contradictorios. En uno (49) se ensayó la administración de ritodrina en cápsulas de liberación prolongada y se observó una reducción significativa de la incidencia del parto pretérmino. El otro estudio (50) se basó en la administración de terbutalina por vía oral, intervención que no produjo efectos diferentes de los del placebo en ninguna de las variables analizadas. Si ello se suma a los datos que muestran la falta de efecto profiláctico de los betamiméticos, la administración de estos agentes por vía oral debería suspenderse para todas las indicaciones actuales, hasta que surja información concluyente que demuestre su utilidad.

Sulfato de magnesio. El uso del sulfato de magnesio con la intención de posponer el parto es uno de tantos ejemplos de intervenciones que se usan en la práctica clínica sin que se hayan evaluado adecuadamente con anterioridad. Hay cuatro ensayos controlados publicados en los que se compara la administración de sulfato de magnesio con la de placebo y la falta de tratamiento. Dos están evaluados en una de las revisiones sistemáticas de la Biblioteca Cochrane (51), en la cual se señala que no hay pruebas de que el sulfato de magnesio posponga el parto o mejore los resultados neonatales. Los otros dos estudios $(52,53)$ fueron publicados después de haber aparecido la revisión. No fueron controlados con placebo e incluyen un total de 155 pacientes en conjunto. El estudio de Fox et al. (52), realizado en embarazadas con 34 a 37 semanas de gestación, no muestra mejores resultados maternos ni neonatales. El otro estudio, de Ma (53), muestra una menor incidencia de parto pretérmino con el uso del sulfato de magnesio. Ninguno de los cuatro estudios tiene una calidad metodológica que lo proteja de sesgos, por lo que no puede inferirse ningún resultado concluyente.

Los estudios que comparan el sulfato de magnesio con los betamiméticos se evalúan en otra revisión de la Biblioteca Cochrane (54). Son de calidad metodológica cuestionable debido a contaminación (las pacientes en las que fracasó el tratamiento con sulfato de magnesio recibieron betamiméticos y viceversa) y a tratamientos de mantenimiento similares en ambos grupos, por lo que no puede realizarse ninguna comparación válida entre las intervenciones. De los seis estudios incluidos, solo uno presenta resultados neonatales. La acumulación de magnesio en el organismo fetal puede, sin duda, producir efectos adversos y, si se considera que el primer objetivo de prevenir el parto pretérmino es mejorar el desenlace neonatal, aquellos estudios que no lo evalúan son inaceptables. Desde que apareció la revisión se han publicado dos estudios más que comparan esas intervenciones $(55,56)$. Ambos adolecen de problemas metodológicos similares a los anteriores. En conclusión, hasta el momento, no hay documentación científica válida que apoye el uso del sulfato de magnesio para tratar la amenaza de parto pretérmino.

Inhibidores de la síntesis de prostaglandinas. Las prostaglandinas tienen una función preponderante en el 
desencadenamiento y mantenimiento del trabajo de parto, por lo que parece lógico inhibir su síntesis para detener el parto. El fármaco más ampliamente utilizado para ese fin es la indometacina. Los ensayos clínicos controlados han demostrado su efectividad para evitar el parto pretérmino en las 48 horas posteriores al inicio del tratamiento y también en los 7 a 10 días después de iniciado el tratamiento. Asimismo, reduce la incidencia de recién nacidos con peso menor de 2500 $\mathrm{g}$ y genera pocos efectos adversos en la madre, sobre todo cuando se administra por vía rectal (57).

El problema con la indometacina es que puede producir efectos adversos en el feto, que van desde la constricción del conducto arterioso hasta efectos sobre la función plaquetaria y la hemodinámica de varios lechos esplácnicos, especialmente el renal. Ninguna de las investigaciones mencionadas previamente demostró estos efectos, pero un estudio observacional de buena calidad publicado en 1993 (58) muestra que el uso de indometacina se asocia con una mayor incidencia de enterocolitis necrotizante, alteraciones del flujo renal, hemorragia endocraneana y cierre prematuro del conducto arterioso en neonatos prematuros con edades gestacionales de menos de 30 semanas. Por lo tanto, se recomienda restringir su uso a períodos de tratamiento no mayores de 48 horas (2).

El reconocimiento de dos tipos diferentes de ciclooxigenasa, una constitutiva y la otra inducible, y la hipótesis de que la segunda pueda estar vinculada con los procesos de inicio del trabajo de parto, abren la puerta al empleo de nuevos medicamentos de este tipo que tengan menos efectos adversos sobre el feto (2).

Bloqueadores de los canales del calcio: nifepidina. Se cree que la acción de los bloqueadores de los canales del calcio se basa en la relajación de la fibra miometrial, y se han publicado informes de varios ensayos controlados sobre su uso (principalmente de la nifepidina) en la literatura médica. La Biblioteca Cochrane recopiló nueve in- vestigaciones en las que se compara la eficacia de dichos bloqueadores con la de los betamiméticos, pero en la revisión sistemática se incluyeron solamente tres estudios por la calidad inaceptable de los restantes. Aunque la nifepidina mostró un efecto inhibidor de las contracciones uterinas comparable al de los betamiméticos, por ahora no se ha demostrado su superioridad y no se conocen en detalle sus posibles efectos adversos. Por ello, su uso debe seguirse restringiendo al contexto de los ensayos clínicos controlados (59).

\section{Disminución de la morbilidad y mortalidad neonatales}

Antibióticos. Como se comentó anteriormente, está documentada la asociación entre la infección latente de las membranas ovulares y el parto pretérmino. Por esta razón se ha intentado la terapia con antibióticos en los casos de amenaza de parto pretérmino con o sin rotura de membranas, con el fin de disminuir la incidencia de premadurez neonatal y la morbilidad y mortalidad neonatales. Las investigaciones publicadas diferencian el efecto de los antibióticos de acuerdo con la integridad de las membranas. Se comentará el estado actual de las evaluaciones por separado, para los casos con membranas íntegras y los casos con membranas rotas.

Efecto con membranas integras. King y Flenady revisaron el tema para la Biblioteca Cochrane (60). La revisión sistemática incluye ocho artículos de buena a muy buena calidad metodológica, siete de ellos controlados con placebo. Los antibióticos probados fueron ampicilina (con o sin inhibidores de la betalactamasa), eritromicina $\mathrm{u}$ otros macrólidos, cefalosporinas, metronidazol y clindamicina, solos o en combinación. En el análisis de los resultados maternos no se observó ningún efecto sobre la prolongación del embarazo y sí una reducción significativa, de $38 \%$, de la infección materna (RR: 0,62; IC95\%: 0,42 a 0,92). Los resultados referentes a los neonatos no mostraron ningún efecto sobre la incidencia de sepsis neonatal, el síndrome de dificultad respiratoria ni la hemorragia intraventricular; pero sí una probable reducción de la enterocolitis necrotizante (RR: 0,39; IC95\% 0,14 a 1,06), y un probable aumento de la mortalidad perinatal (RR: 2,64; IC95\% 0,86 a 8,11).

En otra revisión sistemática reciente (61) se evaluó específicamente el efecto de los antibióticos en los resultados neonatales, que coinciden con los de la revisión de Cochrane. En vista de ello, no se recomienda la administración rutinaria de antibióticos a pacientes con amenaza de parto pretérmino y membranas íntegras.

Efecto con membranas rotas. La rotura prematura de membranas a edades gestacionales tempranas se acompaña del parto pretérmino de manera casi sistemática. Por ello se intenta disminuir mediante el uso de antibióticos la morbilidad y la mortalidad maternas, fetales y neonatales asociadas con este cuadro clínico. Los riesgos de infección provienen en su mayor parte de la entrada de gérmenes en la cavidad amniótica, aunque en algunos casos la infección preceda a la rotura de las membranas. En una revisión sistemática de la Biblioteca Cochrane (62) se analizan 12 investigaciones. En ellas se observa una disminución significativa de las incidencias de parto dentro de los 7 días después de iniciado el tratamiento (RR: 0,82; IC95\%: 0,74 a 0,89), y de las infecciones materna (RR: 0,68; IC95\%: 0,56 a 0,83) y neonatal (RR: 0,72; IC95\%: 0,57 a 0,90).

Los resultados son coherentes y parecen demostrar algún efecto sobre esas variables, por lo que se esperaría una reducción de la mortalidad perinatal. Sin embargo, ese efecto no se observó, lo que deja abierta la posibilidad de que la intervención genere en los fetos o neonatos algún efecto adverso que neutralice el efecto beneficioso sobre las demás variables. Una explicación posible sería que los antibióticos produzcan una mayor sensibilidad a infecciones por otros microorganismos resistentes.

Para evaluar el verdadero efecto de los antibióticos sobre la mortalidad pe- 
rinatal, se realiza un ensayo controlado multicéntrico denominado ORACLE, coordinado en el Reino Unido, que incluirá a 10000 pacientes en riesgo de parto pretérmino con y sin membranas íntegras. Los antibióticos ensayados son amoxicilina más ácido clavulánico y eritromicina. Para fines de septiembre de 1998, se habían seleccionado más de 6000 pacientes y la selección se terminará a principios del año 2000. Argentina es el único país latinoamericano que participa en la investigación.

Prevención del síndrome de dificultad respiratoria neonatal. En esta sección comentaremos dos intervenciones frecuentemente evaluadas, que se aplican para acelerar la maduración pulmonar fetal. Ambas se aplican a embarazadas en alto riesgo de parto pretérmino por diversas causas, entre las 24 y 34 semanas de edad gestacional.

Administración prenatal de corticoides a la embarazada. Esta es sin duda una de las intervenciones de perinatología que más extensa y adecuadamente se han evaluado. En este sentido, se recomienda en especial la lectura de la muy completa revisión sistemática realizada por Crowley (63, 64). En 1994 los Institutos Nacionales de Salud (NIH) de los Estados Unidos organizaron una conferencia de consenso sobre la corticoterapia prenatal (65). He aquí las conclusiones de esa conferencia:

La terapia prenatal con corticoides reduce la mortalidad neonatal, el síndrome de dificultad respiratoria y la hemorragia intraventricular. Estos beneficios se verifican en una amplia gama de edades gestacionales que abarcan desde la 24. ${ }^{\text {a }}$ hasta la 34. ${ }^{\text {a }}$ semana y no se hallan limitados por la raza o el sexo del neonato. Aunque los beneficios máximos de los corticosteroides se alcanzan después de las 24 horas de haber comenzado el tratamiento, aun un tratamiento de menos duración puede mejorar el pronóstico. Los beneficios de los corticosteroides antenatales complementan a los del surfactante pulmonar exógeno. Cuando hay rotura prematura de membranas pretérmino, los corticosteroides producen los mismos efectos beneficiosos que se observan en los casos con membranas íntegras, pero en menor grado. No está claro si este tratamiento incrementa el riesgo de infección en la madre o el neonato. De todas maneras, el riesgo de hemorragia intraventricular o muerte por premadurez es mayor que el riesgo por infección. Los resultados de ensayos clínicos aleatorizados con seguimiento de los niños hasta los 12 años de edad indican que los corticosteroides prenatales no producen efectos adversos en el desarrollo físico ni psicomotor (66).

Administración de hormona liberadora de tirotropina. Los ensayos de Liggins et al. (67) demostraron que la hormona liberadora de tirotropina (TRH) potenciaba los efectos de los corticoides en fetos de oveja. Este descubrimiento generó en la comunidad médica la esperanza de que el escaso efecto de los corticoides en edades gestacionales muy tempranas pudiera solucionarse con la adición de TRH. En consecuencia, se realizaron varios ensayos clínicos, pero casi todos tenían problemas de diseño que no hacían fiables los resultados (68).

En 1995 Crowther publicó los resultados del estudio colaborativo australiano ACTOBAT (69). Con alrededor de 1200 pacientes, es el estudio más grande que se ha efectuado sobre el tema y reúne más participantes que todos los estudios anteriores en conjunto. Los resultados no fueron alentadores: la incidencia del síndrome de dificultad respiratoria neonatal es llamativamente mayor en el grupo tratado con TRH más corticoides que en el grupo tratado con placebo más corticoides (35\% frente a 30\%). Además, la administración de TRH se asoció con una alta incidencia de efectos adversos (20\%). En el transcurso de 1998 se han publicado dos nuevas investigaciones colaborativas $(70,71)$ que evaluaron la administración de TRH sumada a corticoides. Ambos estudios en conjunto incluyeron a 1300 pacientes y los resultados coinciden con los del estudio australiano en que no hubo efecto beneficioso sobre ningún resultado neonatal clínicamente importante. En vista de ello, la administración de TRH sumada a la de corticoides no está actualmente indicada para prevenir la morbilidad neonatal.

\section{CONCLUSIONES}

En esta revisión hemos analizado rigurosamente los datos científicamente validados disponibles en la literatura sobre métodos que permitan predecir, prevenir o detectar precozmente la amenaza de parto pretérmino y los tratamientos que puedan detener esa amenaza o significar un mejor desenlace para las embarazadas que experimentan el parto pretérmino. De nuestra revisión se desprende que, a pesar de haberse evaluado una larga serie de métodos y tratamientos, son muy pocos los que han mostrado su efectividad y merecen implementarse en la práctica clínica.

En relación con la detección precoz del parto pretérmino, puede concluirse que la selección de factores de riesgo, la educación de las embarazadas para que aprendan a detectar los signos de alarma, el examen vaginal seriado y el monitoreo domiciliario de la actividad uterina no han manifestado ser métodos fiables que justifiquen su incorporación en la práctica clínica. La medición de la fibronectina es un método promisorio pero aún se requieren estudios que comprueben su eficiencia y justifiquen su uso.

Con respecto a la prevención del parto pretérmino, son pocas las intervenciones con algún beneficio comprobado y su uso es muy limitado. El tamizaje de la bacteriuria asintomática y su tratamiento reducen la incidencia del parto pretérmino y deben formar parte de todos los programas de control prenatal. Asimismo, el tamizaje de la vaginosis bacteriana y su tratamiento reducen la incidencia del parto pretérmino, pero solo en aquellas mujeres que tienen antecedentes de partos prematuros. El cerclaje profiláctico también es efectivo solo en mujeres con antecedentes de tres o más partos pretérmino. 
En lo que respecta al tratamiento de la amenaza de parto pretérmino, la aplicación de betamiméticos puede prolongar el período de latencia (desde el inicio del tratamiento al parto) por 48 horas. Lo atractivo de esta opción es que permite aplicar a la madre las dosis preconizadas de corticosteroides. No se han obtenido beneficios del uso más prolongado de betamiméticos. La indometacina prolonga el período de latencia, aunque no se recomienda como tratamiento de primera elección por sus posibles efectos sobre el feto. Como se ha hecho notar, en los casos de rotura prematura de las membranas, los antibióticos son útiles para prolongar el período de latencia.

Como es bien sabido, la administración de corticosteroides a la madre disminuye la incidencia del síndrome de dificultad respiratoria neonatal, lo que implica una mayor supervivencia neonatal. Su uso debe difundirse en todas partes.

En el cuadro 2 se presentan las intervenciones evaluadas de acuerdo con las pruebas de su eficacia.

\section{Áreas que requieren investigación}

Dada la gran importancia que tienen para la salud pública de la Región de las Américas los problemas aquí expuestos, deben continuarse y apoyarse los proyectos de investigación pertinentes. Hasta la fecha los resultados han sido más bien frustrantes, lo que se explica por el hecho de que hay muy pocos conocimientos sobre los mecanismos que provocan el inicio del parto y por qué actúan prematuramente en determinadas mujeres. Es necesario generar nuevas hipótesis etiológicas y realizar más investigaciones básicas y epidemiológicas sobre las causas y la prevención de los partos pretérmino. Mientras tanto, no es de esperar que se reduzcan las incidencias de este trastorno en los países en desarrollo ni en los desarrollados.

Sobre la base de lo conocido hasta la fecha, cabe considerar el tratamiento de la vaginosis bacteriana como método profiláctico en poblaciones nece-

\section{CUADRO 2. Intervenciones evaluadas de acuerdo con sus efectos}

Intervenciones beneficiosas

Tamizaje de bacteriuria asintomática durante el embarazo y tratamiento con antibióticos para reducir la incidencia de parto pretérmino

Tamizaje de vaginosis bacteriana en embarazadas con antecedentes de parto pretérmino para reducir la incidencia de parto pretérmino

Cerclaje cervical profiláctico de embarazadas con antecedentes de tres o más partos pretérmino para reducir la incidencia de parto pretérmino

Tratamiento de la amenaza de parto pretérmino con betamiméticos por vía parenteral para posponer el parto por un período de 24 a $48 \mathrm{~h}$

Administración de antibióticos a la embarazada en casos de rotura prematura de membranas para reducir la morbilidad neonatal y materna

Administración de corticoides a la embarazada para reducir la mortalidad perinatal, la incidencia del síndrome de dificultad respiratoria y la hemorragia intraventricular neonatales

Intervenciones probablemente beneficiosas, pero con algún efecto adverso importante

Tratamiento de la amenaza de parto pretérmino con inhibidores de la síntesis de prostaglandinas (indometacina) para posponer el parto por 24 a $48 \mathrm{~h}$, pero con posibles efectos adversos graves sobre el neonato

Intervenciones con pruebas inconcluyentes o contradictorias sobre su efectividad

Predicción del parto pretérmino mediante la detección de fibronectina fetal en el flujo cervical

Administración a embarazadas malnutridas de suplementos calórico-proteicos para reducir la incidencia de parto pretérmino

Suplementación nutricional de las embarazadas con deficiencia de calcio, magnesio, cinc o hierro y folatos, para reducir la incidencia de parto pretérmino

Administración de progesterona durante el embarazo para reducir la incidencia de parto pretérmino Tratamiento de la amenaza de parto pretérmino con bloqueadores de los canales del calcio para posponer el parto

Tratamiento de la amenaza de parto pretérmino con sulfato de magnesio para posponer el parto

Administración a la embarazada de betamiméticos por vía oral como mantenimiento luego de haberse detenido la amenaza de parto pretérmino

Intervenciones probablemente inefectivas

Selección de pacientes embarazadas según factores de riesgo para programas de reducción del parto pretérmino

Educación de las embarazadas para que reconozcan los signos de alarma

Monitoreo de la actividad uterina en el hogar

Examen vaginal periódico para detectar precozmente la amenaza de parto pretérmino

Apoyo social durante el embarazo

Administración profiláctica de betamiméticos por vía oral

Cerclaje cervical durante el embarazo de mujeres sin o con pocos antecedentes de partos pretérmino

Administración de antibióticos a embarazadas con amenaza de parto pretérmino y membranas íntegras

Administración de hormona liberadora de tirotropina a la embarazada para inducir la madurez pulmonar fetal

sitadas en las que es factible hallar prevalencias más altas de la infección en embarazadas que pudieran beneficiarse con este tratamiento. También es de interés investigar métodos para predecir el parto pretérmino $\mathrm{y}$, entre ellos, los marcadores bioquímicos como la fibronectina $\mathrm{u}$ otros recientemente propuestos, como la hormona liberadora de corticotropina placentaria $(\mathrm{CRH})$.

Si bien se ha ensayado la administración de una serie de suplementos nu- tricionales (proteínas, calorías, hierro, folatos, cinc, calcio) a las embarazadas en riesgo, no se ha observado que tengan efectos concluyentes en la reducción del parto pretérmino. No obstante, deben seguirse explorando los posibles efectos de micronutrientes, sobre todo en poblaciones con carencias nutricionales.

Para el tratamiento del parto pretérmino ya iniciado, los nuevos esquemas de administración de betamiméticos, los inhibidores de la síntesis de prosta- 
1. Villar J, Ezcurra EJ, Gurtner de la Fuente V, Campodónico L. Pre-term delivery syndrome: the unmet need. Research \& Clinical Forums 1994; 16: 9-33.

2. Keirse MJNC. New perspectives for the effective treatment of preterm labor. Am J Obstet Gynecol 1995;173:618-628.

3. Rogowski JA. The economics of preterm delivery. Prenat Neonat Med 1998;3:16-20.

4. Chalmers I, Altman D. Foreword. En: Chalmers I, Altman DG, eds. Systematic reviews. London: BMJ Publishing Group; 1995.

5. Pregnancy and Childbirth Module. En: The Cochrane Database of Systematic Reviews [base de datos en disquete $\mathrm{y}$ CDROM]. Oxford: The Cochrane Collaboration; Issue 2, 1995. Update Software. Puede conseguirse de BMJ Publishing Group, Londres.

6. The Cochrane Library. The Cochrane Library Database on Disk and CDROM. Oxford: The Cochrane Collaboration, Issue 3, 1998. Update Software.

7. Alexander S, Keirse MJNC. Formal risk scoring during pregnancy. En: Chalmers I, Enkin M, Keirse MJNC. Effective care in pregnancy and childbirth. Oxford University Press: 1989

8. McLean M, Walters WAW, Smith R. Prediction and early diagnosis of preterm labor: a critical review. Obstet Gynecol Survey 1993;48:209-225.

9. Hueston WJ, Knox MA, Eilers G, Pauwels J, Lonsdorf D. Education in the prevention of preterm birth. Obstet Gynecol 1995:86:705-712.

10. Buekens P, Alexander S, Boutsen M, Blondel B, Kaminski M, Rejd M, and European Community Collaborative Study Group on Prenatal Screening. Randomised controlled trial on routine cervical examination in pregnancy. Lancet 1994;344: 841-844.

11. Collaborative Home Uterine Monitoring Study (CHUMS) Group. A multicenter randomized controlled trial of home uterine monitoring: active versus sham device. Am J Obstet Gynecol 1995;173: 1120-1127.

12. Dyson DC, Danbe KH, Bamber JA, et al. Monitoring women at risk for preterm labor. N Eng J Med 1998; 338: 15-19.

13. Lockwood CJ, Senyei AE, Dische MR, Casal D, Shah KD, Thung SN, et al. Fetal fibronectin in cervical and vaginal secretions as a predictor of preterm delivery. $\mathrm{N}$ Eng J Med 1991;325:669-674.

14. Chien PFW, Khan K, Ogston S, Owen P. The diagnostic accuracy of cervico-vaginal fetal fibronectin in predicting preterm delivery: an overview. Br J Obstet Gynaecol 1997;104:436-444.

15. Faron $G$, Boulvain $M$, Irion $O$, Bernard PM, Fraser WD. Prediction of preterm delivery by fetal fibronectin: a meta-analysis. Obstet Gynecol 1998;92:153-158.
16. Hodnett ED. Support from caregivers during at-risk pregnancy [revisado el 20 de diciembre de 1994]. En: Keirse MJNC, Renfrew MJ, Neilson JP, Crowther C, eds. Pregnancy and childbirth module. En: The Cochrane Database of Systematic Reviews [base de datos en disquete y CDROM]. Oxford: The Cochrane Collaboration; Issue 2, 1995. Update Software. Puede conseguirse de BMJ Publishing Group, Londres.

17. MRC/RCOG Working Party on Cervical Cerclage. Final report of the Medical Research Council/Royal College of Obstetricians and Gynaecologists multicentre randomized trial of cervical cerclage. $\mathrm{Br} \mathrm{J}$ Obstet Gynaecol 1993;100:516-523.

18. Keirse MJNC. Prophylactic oral betamimetics in pregnancy [revisado el 2 de abril de 1992]. En: Keirse MJNC, Renfrew MJ, Neilson JP, Crowther C, eds. Pregnancy and childbirth module. En: The Cochrane Pregnancy and Childbirth Database [base de datos en disquete y CDROM]. Oxford: The Cochrane Collaboration; 1995. Issue 2, Update Software. Puede conseguirse de BMJ Publishing Group, Londres.

19. Kramer MS. Determinants of low birth weight: methodological assessment and meta-analysis. Bull World Health Org 1987;65(5):663-737.

20. Kramer MS. Maternal nutrition, pregnancy outcome and public health policy. CMAJ 1998;159:663-665.

21. A WHO Collaborative Study of Maternal Anthropometry and Pregnancy Outcomes. Int J Gynecol Obstet 1997;57:1-15.

22. Kramer MS. Balanced protein/energy supplementation in pregnancy (Cochrane Review). En: Oxford: The Cochrane Library, Issue 3, 1998. Update Software.

23. Kramer MS. High protein supplementation in pregnancy (Cochrane Review). En: Oxford: The Cochrane Library, Issue 3; 1998. Update Software.

24. Kramer MS. Isocaloric balanced protein supplementation in pregnancy (Cochrane Review). En: Oxford: The. Update Software.

25. Ceesay SM, Prentice AM, Cole TJ, et al. Effects on birth weight and perinatal mortality of maternal dietary supplements in Rural Gambia: a 5 year randomised controlled trial. BMJ 1997;315:786-790.

26. Sibai BM, Villar MA, Bary E. Magnesium supplementation during pregnancy: a double-blind randomized controlled clinical trial. Am J Obstet Gynecol 1989; 161:115-119.

27. Makrides M, Crowther CA. Magnesium supplementation during pregnancy (Cochrane Review). En: Oxford: The Cochrane Library, Issue 3; 1998. Update Software.

28. Carroli G, Duley G, Belizán JM, Villar J. Calcium supplementation during pregnancy: a sytematic review of randomized controlled trials. Br J Obstet Gynaecol 1994;101:753-758.

29. Bucher HC, Guyatt GH, Cook RJ, Hatala R, Cook DJ, Lang JD, et al. Effect of cal- cium supplementation on pregnancyinduced hypertension and preeclampsia: a meta-analysis of randomized controlled trials. JAMA 1996; 275 (14):1113-1117.

30. Atallah AN, Hofmeyr GJ, Duley L. Calcium supplementation during pregnancy to prevent hypertensive disorders and related adverse outcomes (Cochrane Review). En: Oxford: The Cochrane Library, Issue 3; 1998. Update Software.

31. Kiilholma P, Gronroos M, Erkkola P, et al. The role of calcium, iron, copper and zinc in preterm delivery and premature rupture of membranes. Gynecol Obstet Invest 1984;17:194-201.

32. Mahomed K. Zinc supplementation in pregnancy (Cochrane Review). En: Oxford: The Cochrane Library, Issue 3; 1998. Update Software.

33. Goldenberg RL, Tamura T, Neggers $Y$ Cooper RL, Johnston KE, DuBard MB, et al. The effect of zinc supplementation on pregnancy outcome. JAMA 1995;274: 463-468.

34. Jonsson B, Hauge B, Larsen MF, et al. Zinc supplementation during pregnancy: a double blind randomized controlled trial. Acta Obstet Gynecol Scand 1996;75: 725-729.

35. Villar J, Gülmezoglu M, de Onis M. Nutritional and antimicrobial interventions to prevent preterm birth: an overview of randomized controlled trials. Obstet Gynecol Survey 1998;53:575-585.

36. Mahomed K. Routine iron supplementation during pregnancy (Cochrane Review). En: Oxford: The Cochrane Library, Issue 3; 1998. Update Software.

37. Mahomed K. Routine folate supplementation in pregnancy (Cochrane Review). En: Oxford: The Cochrane Library, Issue 3, 1998. Update Software.

38. Prendiville WJ. Progestogens to prevent miscarriage and preterm birth. [revisado el 17 de septiembre de 1993]. En: Keirse MJNC, Renfrew MJ, Neilson JP, Crowther C, eds. Pregnancy and childbirth module. En: The Cochrane Pregnancy and Childbirth Database [base de datos en disquete y CDROM]. Oxford: The Cochrane Collaboration; Issue 2; 1995. Update Software. Puede conseguirse de BMJ Publishing Group, Londres.

39. Smaill F. Antibiotic vs no treatment for asymptomatic bacteriuria in pregnancy (Cochrane Review). En: Oxford: The Cochrane Library, Issue 3; 1998. Update Software.

40. Hay PE, Lamont RF, Taylor-Robinson D, Morgan DJ, Ison C, Pearson J. Abnormal bacterial colonisation of the genital tract and subsequent preterm delivery and late miscarriage. BMJ 1994;308:295-298.

41. Hillier S, Nugent RP, Eschenbach DA, Krohn MA, Gibbs RS, Martin DH, et al. Association between bacterial vaginosis and preterm delivery of a low-birthweight infant. N Eng J Med 1995;333: 1737-1742. 
42. McDonald HM, O'Loughlin JA, Vigneswaran R, Jolley PT, McDonald PJ. Bacterial vaginosis in pregnancy and efficacy of short-course oral metronidazole treatment: a randomized controlled trial. $\mathrm{Ob}-$ stet Gynecol 1994;84:343-348.

43. Hauth JC, Goldenberg RL, Andrews WW, DuBard MB, Copper RL. Reduced incidence of preterm delivery with metronidazole and erythromycin in women with bacterial vaginosis. N Eng J Med 1995;333: 1732-1736.

44. Brocklehurst $\mathrm{P}$, Hannah M, McDonald $\mathrm{H}$. The management of bacterial vaginosis in pregnancy (Cochrane Review). En: Oxford: The Cochrane Library, Issue 4: 1998. Update Software.

45. Canadian Preterm Labor Investigators Group. Treatment of preterm labor with the beta-adrenergic agonist ritodrine. $\mathrm{N}$ Eng J Med 1992;327:308-312.

46. Keirse MJNC. Betamimetic tocolytics in preterm labour. [revisado el 17 de febrero de 1993]. En: Keirse MJNC, Renfrew MJ, Neilson JP, Crowther C, eds. Pregnancy and childbirth module. En: The Cochrane Pregnancy and Childbirth Database [base de datos en disquete y CDROM]. Oxford: The Cochrane Collaboration, Issue 2; 1995. Update Software. Puede conseguirse de BMJ Publishing Group, Londres.

47. Holleboom CAG, Merkus JMWM, Van Elferen LWM, Keirse MJNC. A loading model for ritodrine administration in preterm labour. Br J Obstet Gynaecol 1993; 100:1107-1110.

48. Macones GA, Berlin M, Berlin J. Efficacy of oral beta-agonist maintenance therapy in preterm labor: A meta-analysis. Obstet Gynecol 1995;85:313-317.

49. Holleboom CA, Merkus JM, va Elferen LW, Keirse MJ. Double-blind evaluation of ritodrine sustained release for oral maintenance of tocolysis after active preterm labour. Br J Obstet Gynaecol 1996; 103:702-705.

50. Lewis R, Mercer BM, Salama M, Walsh MA, Sibai BM. Oral terbutaline after parenteral tocolysis: a randomized, doubleblind placebo-controlled trial. Am J Obstet Gynecol 1996;175:834-837.

51. Keirse MJNC. Magnesium sulphate in preterm labour. [revisado el 2 de abril de 1992]. En: Keirse MJNC, Renfrew MJ, Neilson JP, Crowther C, eds. Pregnancy and childbirth module. En: Oxford: The Cochrane Pregnancy and Childbirth Database [base de datos en disquete $\mathrm{y}$ CDROM]. Oxford: The Cochrane Collaboration, Issue 2; 1995. Update Software. Puede conseguirse de BMJ Publishing Group, Londres.
52. Fox MD, Allbert JR, McCaul JF, Martin RW, McLaughlin BN, Morrison JC. Neonatal morbidity between 34 and 37 weeks' gestation. J Perinatol 1993;13:349-353.

53. Ma L. Magnesium sulfate in prevention of preterm labor. Chung-Hua-I-Hsueh-TsaChih-Taipei 1992;72:158-161.

54. Keirse MJNC. Magnesium sulphate vs betamimetics for tocolysis in preterm labour. [revisado el 22 de abril de 1993]. En: Keirse MJNC, Renfrew MJ, Neilson JP, Crowther C, eds. Pregnancy and childbirth module. En: The Cochrane Pregnancy and Childbirth Database [base de datos en disquete y CDROM]. Oxford: The Cochrane Collaboration; Issue 2; 1995. Update Software. Puede conseguirse de BMJ Publishing Group, Londres.

55. Chau AC, Gabert A, Miller JM. A prospective comparison of terbutaline and magnesium for tocolysis. Obstet Gynecol 1992; 80:847-851.

56. Zhu B, Fu Y. Treatment of preterm labor with ritodrine. Chung-Hua-Fu-Chan-KoTsa-Chih 1996;31:721-723.

57. Keirse MJNC. Indomethacin tocolysis in preterm labour. [revisado el 14 de agosto de 1992]. En: Keirse MJNC, Renfrew MJ, Neilson JP, Crowther C, eds. Pregnancy and childbirth module. En: The Cochrane Pregnancy and Childbirth Database [base de datos en disquete y CDROM]. Oxford: The Cochrane Collaboration; Issue 2; 1995. Update Software. Puede conseguirse de BMJ Publishing Group, Londres.

58. Norton ME, Merrill J, Cooper BAB, Kuller JA, Clyman RI. Neonatal complications after the administration of indomethacin for preterm labor. N Eng J Med 1993;329: 1602-1607.

59. Keirse MJNC. Calcium antagonists vs betamimetics in preterm labour. [revisado el 22 de abril 1993] En: Keirse MJNC, Renfrew MJ, Neilson JP, Crowther C, eds. Pregnancy and childbirth module. En: The Cochrane Pregnancy and Childbirth Database [base de datos en disquete y CDROM]. Oxford: The Cochrane Collaboration, Issue 2; 1995. Update Software. Puede conseguirse de BMJ Publishing Group, Londres.

60. King J, Flenady V. Antibiotics in preterm labour with intact membranes (Cochrane Review). En: The Cochrane Library, Issue 3; 1998. Update Software.

61. Egarter C, Leitich H, Husslein P, Kaider A, Schemper M. Adjunctive antibiotic treatment in preterm labor and neonatal morbidity: A meta-analysis. Obstet Gynecol 1996;88:303-309.

62. Kenyon S, Boulvain M. Antibiotics for preterm premature rupture of mem- branes (Cochrane Review). En: Oxford: The Cochrane Library, Issue 3; 1998. Update Software.

63. Crowley P. Corticosteroids prior to preterm delivery. [revisado el 19 de agosto 1994] En: Keirse MJNC, Renfrew MJ, Neilson JP, Crowther C, eds. Pregnancy and childbirth module. En: The Cochrane Pregnancy and Childbirth Database [base de datos en disquete y CDROM]. Oxford: The Cochrane Collaboration, Issue 2; 1995. Update Software. Puede conseguirse de BMJ Publishing Group, Londres.

64. Crowley P. Antenatal corticosteroid therapy: A meta-analysis of randomized trials, 1972 to 1994. Am J Obstet Gynecol 1995;173:322-335.

65. NIH Consensus Development Panel on the Effect of Corticosteroids for Fetal Maturation on Perinatal Outcomes. Effect of Corticosteroids for Fetal Maturation on Perinatal Outcomes (NIH Consensus Conference). JAMA 1995;273:413-418.

66. Althabe F. Inducción farmacológica de la madurez pulmonar fetal. En Fábregues G, ed. Hipertensión y embarazo. Bayer Argentina 1996.

67. Liggins GC, Schellenberg JC, Manzai M, Kitterman JA, Lee CG. Synergisms of cortisol and thyrotropin releasing hormone in lung maturation in fetal sheep. J App Physiol 1988;65:1880-4

68. Crowther CA, Alfirevic Z, Haslam RR. Antenatal thyrotropin-releasing hormone (TRH) prior to preterm delivery (Cochrane Review). En: Oxford:The Cochrane Library, Issue 3; 1998. Update Software.

69. The ACTOBAT Study Group. Australian collaborative trial of betamethasone and thyrotropin-releasing hormone (ACTOBAT) for the prevention of neonatal respiratory disease. Lancet 1995; 345:877-882.

70. Ballard RA, Ballard PL, Cnaan A, Pinto Martin J, Davis DJ, Padbury JF, et al. Antenatal thyrotropin-releasing hormone to prevent lung disease in preterm infants. American Thyrotropin-Releasing Hormone Study Group. N Eng J Med 1998; 338:493-498.

71. Collaborative Santiago Surfactant Group. Collaborative trial of prenatal thyrotropin-releasing hormone and corticosteroids for prevention of respiratory distress syndrome. Am J Obstet Gynecol 1998;178:33-39.

Manuscrito recibido el 9 de julio de 1997 y aceptado para publicación en versión revisada el 31 de diciembre de 1998. 
ABSTRACT Every year around the world some 13 million premature children are born. Most of these children are born in developing countries, and they account for the largest share of perinatal morbidity and mortality. This review study analyzed scientifically vali-

Preterm delivery: prevention through risk detection and treatment dated data on interventions to prevent at least some portion of these preterm deliveries and to lessen their impact on neonatal health. The Cochrane and MEDLINE bibliographic databases were consulted. Fifty review pieces and research articles were studied, relating to the following aspects of preterm delivery: risk factors and early detection of the risk of preterm delivery; preventing the risk of preterm delivery; treating preterm delivery once it has begun; and preventing neonatal respiratory distress syndrome. There were few successful approaches to the prediction, prevention, or early detection of the threat of preterm delivery. The only measures that can be recommended for all pregnant women are screening for and treating asymptomatic bacteriuria as a part of prenatal check-ups. Screening for bacterial vaginosis and treating it reduce the incidence of preterm births in pregnant women with a history of premature delivery. In addition, prophylactic cerclage decreases the incidence of premature births in pregnant women who have had more than three preterm births. To treat a delivery that starts early, with or without premature membrane rupture, the interventions that have proved to be effective are administering betamimetics to the parturient woman in order to delay delivery for 48 hours, and using indomethacin for the same purpose, as the second-choice drug. The prenatal administration of corticosteroids to the pregnant woman can induce lung maturation in the fetus and reduce respiratory distress syndrome and ventricular hemorrhage, thus decreasing neonatal mortality. There is a need to continue and support basic and epidemiological research in order to develop new knowledge on the causes and mechanisms of preterm delivery and on preventing the morbidity and mortality that preterm delivery produces. 